\title{
COMMUNICATION
}

\section{Apport de l'imagerie CBCT dans l'évaluation des critères radiologiques de bénignité et malignité des tumeurs des maxillaires}

\author{
Moreau $\mathbf{N}^{1,2}$, Lafond $\mathbf{M}^{1}$, Ejeil AL', Salmon $\mathbf{B}^{1,3}$ \\ 1 - Service d'odontologie, Hôpital Bretonneau, AP-HP, Hôpitaux Universitaires Paris Nord Val de Seine, Paris, \\ France
}

2 - INSERM UMR 894, Centre de Psychiatrie et Neurosciences, Université Paris Descartes Sorbonne Paris Cité, Hôpital Sainte-Anne, Paris, France

3 - EA 2496, Université Paris Descartes Sorbonne Paris Cité, Faculté de Chirurgie Dentaire, Montrouge, France

\section{Introduction}

Les tumeurs des maxillaires regroupent un ensemble varié de pathologies osseuses, bénignes ou malignes, de diagnostic souvent délicat. Ces dernières se traduisent - le plus souvent - par une perte de substance dans le cas de lésions kystiques ou ostéolytiques, tandis que d'autres tumeurs peuvent se manifester par la formation d'un tissu minéralisé à l'origine d'une image radio-opaque (Martin-Duverneuil N. 2013).

Le caractère malin de certaines de ces tumeurs mais également le risque de transformation maligne de quelques tumeurs bénignes doivent inciter à la rigueur et à la prudence lors de l'établissement du diagnostic (Sauveur $G$ et al. 2008). Ce dernier est souvent peu aisé face à des aspects cliniques et radiographiques peu spécifiques, surtout en imagerie conventionnelle. À ce titre, l'imagerie tridimensionnelle (TDM ou CBCT) revêt une importance fondamentale dans l'élaboration des hypothèses diagnostiques initiales avant examen anatomo-pathologique.

\section{Matériels et méthodes}

Ce travail propose, à travers une revue narrative de la littérature scientifique, de souligner l'importance d'une analyse radiographique tridimensionnelle fine face à une lésion maxillomandibulaire non diagnostiquée, afin d'évaluer le risque potentiel de malignité, facilitant ainsi la confrontation clinico-radiographique nécessaire au diagnostic final.

\section{Résultats}

L'analyse de la littérature scientifique rapporte - aux travers de nombreuses séries de cas cliniques - différents critères radiographiques évocateurs de bénignité ou de malignité qui peuvent être divisés didactiquement en critères majeurs de bénignité, critères majeurs de malignité et critères non spécifiques (Lafond M. 2014).

\section{Discussion}

La majorité des critères majeurs (tant de bénignité que de malignité) nécessitent une imagerie tridimensionnelle pour leur évaluation radiologique précise. Ainsi, le refoulement des structures avoisinantes ou la soufflure des corticales, tous deux évocateurs de bénignité, mais également la 
destruction corticale et la destruction des structures avoisinantes, signes évocateurs de malignité, ne peuvent être correctement appréhendés qu'en imagerie tridimensionnelle. À ce titre, l'imagerie CBCT est l'examen radiographique de choix en pratique courante de médecine et chirurgie orale.

\section{Conclusion}

L'imagerie $\mathrm{CBCT}$, en permettant une analyse tridimensionnelle fine des lésions maxillomandibulaires et des structures avoisinantes, apporte une aide précieuse dans l'évaluation diagnostique initiale de ces lésions en pratique quotidienne. La connaissance des critères cliniques et surtout radiographiques de bénignité et malignité des lésions des maxillaires doit permettre une amélioration de leur prise en charge et donc, un meilleur pronostic.

\section{Références}

Lafond M. Critères clinico-radiographiques de bénignité et malignité en pathologie des maxillaires. Thèse d'exercice : Chirurgie dentaire : Université Paris Descartes : 2014.

Martin-Duverneuil N. Tumeurs du massif facial, EMC Radiologie et imagerie médicale - 31-675-A-20, 2013.

Sauveur G, Ferkdadji L, Gilbert E, et al. Kystes des maxillaires, EMC Médecine buccale - 28-550-G-10, 2008. 